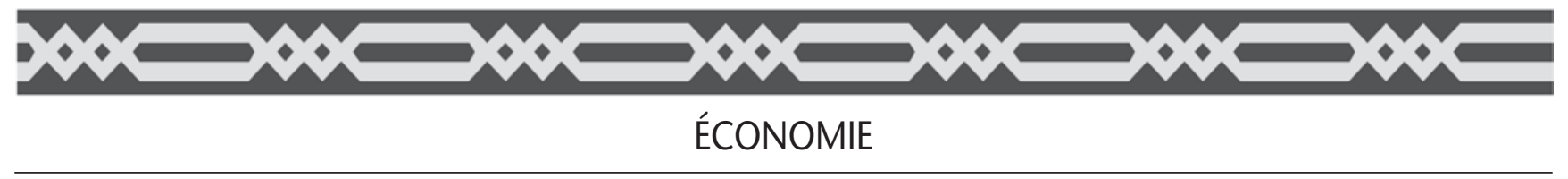

\title{
Pourquoi les prix agricoles augmentent-ils ?
}

\author{
Jean-Marc BOUSSARD ${ }^{1}$ \\ Françoise GÉRARD ${ }^{2}$ \\ Marie-Gabrielle PIKETTY3 \\ ${ }^{1}$ Ancien directeur de recherche INRA ; \\ Membre de l'Académie d'Agriculture, \\ 3 quai Malaquais 75006 Paris \\ <boussard@ivry.inra.fr> \\ ${ }^{2}$ CIRAD, 45bis avenue de la Belle Gabrielle, \\ 94736 Nogent sur Marne \\ <francoise.gerard@cirad.fr> \\ ${ }^{3}$ CIRAD, Université de Sao Paulo, \\ Rua Ferreira Franca 40, Appdo 22A, \\ Alto pinheiro, Sao Paulo, Bresil \\ < piketty@cirad.fr>
}

Partout dans le monde, les prix agricoles augmentent [1], du moins la plupart d'entre eux (le sucre fait exception). Ce ne sont pas des augmentations limitées, de 10 ou $20 \%$, comme celles que prévoyaient les modèles économétriques «d'équilibre général » ou d'équilibre partiel qui ont servi à convaincre les législateurs des bienfaits de la libéralisation (figure 1) ${ }^{1}$. Ce sont des doublements, parfois des triplements, sans aucun rapport avec les coûts de production. Heureusement, cela ne se voit pas (pas encore trop!) sur les prix de I'alimentation dans les pays développés, parce que la matière première n'est souvent qu'une fraction des coûts totaux des « filières ». II faut quand même s'attendre dans ces pays à des répercussions significatives sur les prix de détails, lorsque tous les coûts auront été réajustés. Quant aux pays en voie de développement, où les filières sont plus courtes, et où l'impéritie des dirigeants a conduit à compter sur le marché international pour assurer la sécurité alimentaire, leur situation va bientôt devenir dramatique.

Les questions qui se posent sont alors de savoir pourquoi, si cela va durer, et à qui cela profite.

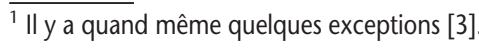

\begin{abstract}
Agricultural prices recently rocketed, calling for significant food price increase, and granting farmers large profits they did not claim. Such an increase in agricultural commodity prices were considered perfectly unrealistic a few years ago. Many explanations are now provided, involving drought in Australia, demand for biofuels, and consumer habits evolution in India or in China. Without denying any validity to such assertions, another hypothesis is proposed here, supported by the ID3 model by CIRAD: the recent price upsurge is only the logical consequence of expectation errors and the unstable character of the market equilibrium point. In this context, the deregulation of markets, particularly by the EC (complying with WTO requirements), which, till recently, was the ultimate stockpile operator, created a new situation likely to feed a chaotic cobweb. This implies that the high price episode will only be temporary, and followed by even more depressed prices. Yet, it might happen that the increased volatility leads to an average price higher than during the state involvement, contrary to policy makers expectations.
\end{abstract}

JEL classification: D4 (market structure and pricing), D5 (general equilibrium and disequilibrium, E3 (Cycle forecasting and simulating), Q1 (Agriculture)

Key words: Agricultural prices

\section{Pourquoi ?}

\section{Les explications "standard »}

On a accusé les spéculateurs, ainsi que la sécheresse en Australie, les biocarburants [2], le développement de la Chine et de l'Inde.

II est vrai que l'offre australienne de céréales s'est considérablement réduite depuis quelques années. Mais est-ce le fait de la sécheresse, ou du calcul économique des agriculteurs en réponse aux baisses de prix, ou même au fait que de nombreux ouvrages d'irrigation ont été démantelés ? Curieusement, il y a eu en 2007 en Argentine une sécheresse sans doute plus réellement importante que la sécheresse australienne de 2006, parce qu'elle intéressait des zones à forts rendements, au lieu que la sécheresse australienne concernait en bonne part des zones désertiques. Pourtant, personne n'en a parlé!

Il est vrai aussi que s'est développée en Chine et en Inde une nouvelle classe sociale «bourgeoise ", qui adopte un mode de vie occidental, avec une consommation par tête de calo-

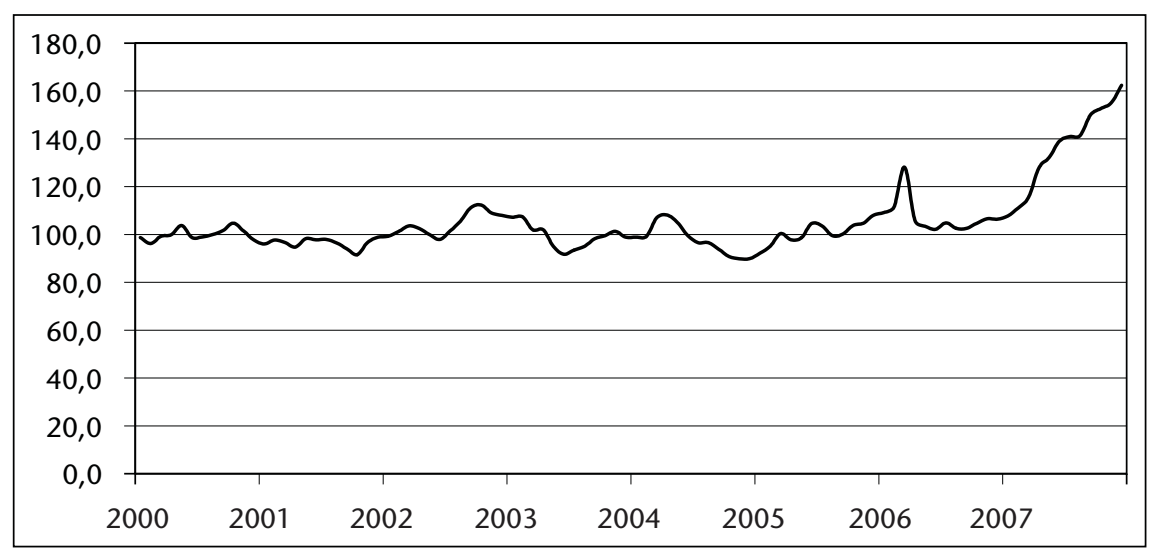

Figure 1. Indice des prix des matières premières alimentaires importées en France (en euros, source Agreste). 
ries directes et indirectes ${ }^{2}$ fortement accrue. Cela évidemment augmente la demande solvable, et pèse sur les marchés. II est tout aussi certain que la demande d'agrocarburants s'est accrue du fait de l'accroissement des prix du pétrole, diminuant ipso facto l'offre agricole, et entraînant donc logiquement une augmentation des prix, toutes choses égales d'ailleurs.

Est-ce à dire cependant que ces explications sont satisfaisantes? Elles sont pourtant assez courtes en face de l'ampleur du phénomène. Aucun des nombreux modèles économétriques « standard » en service actuellement n'a jamais envisagé des variations de prix aussi importantes, en dépit de leurs efforts pour montrer que, justement, grâce à la loi des grands nombres (s'il est possible que la sécheresse fasse baisser la production en Australie, il est presque sûr que l'on aura des récoltes meilleures que prévu quelque part ailleurs dans le monde), la libéralisation allait permettre d'atténuer les « chocs aléatoires».

II y a peu, en 2005, de nombreux auteurs, pourtant conscients de la possibilité de tels phénomènes, parlaient volontiers à la suite de Prebisch [5] et de Singer [6] d'une sorte de « loi d'airain » historique qui impose la baisse séculaire des cours des matières premières agrico$l s^{3}$. Les projections (pourtant généralement optimistes) du modèle FAPRI prévoyaient aussi des évolutions très "plates» des principaux produits agricoles (figure 2), même si elles étaient croissantes. De nombreux autres modèles envisageaient jusqu'à une date récente des situations du même genre. Ils n'intégraient pas les sécheresses, ni les biocarburants, mais tenaient compte de la croissance en Chine et en Inde. Ils se sont lourdement trompés. Est-ce à dire que la sécheresse et les agrocarburants à eux seuls sont responsables du changement de régime actuel ? Ou faut-il trouver autre chose? Un modèle alternatif construit au Cirad ${ }^{4}$. il y a quelques années permet en tout cas de jeter un

\footnotetext{
${ }^{2}$ On sait que la consommation alimentaire traditionnelle en Inde et en Chine est axée sur les calories d’origine végétale, ce qui est « économe » par rapport aux schémas de consommation alimentaire européens ou américains, fondés sur des calories « animales ». II faut en effet 5 à 7 calories « végétales » pour obtenir une calorie « animale ».

${ }^{3}$ Voir par exemple l'articler de Hugon dans l'ouvrage collectif édité par Boussard et Delorme [7] : « Les prix des matières premières agricoles sont fluctuants, avec, semble-t-il, une tendance à la baisse dans le long terme. " De même Géronimi [8] dans le même ouvrage: «Les prix réels baissent-ils à long terme? Oui, mais faiblement »..... on pourrait multiplier les citations de ce genre. Encore les collaborateurs de cet ouvrage étaient-ils très prudents !

${ }^{4}$ Centre international de recherches pour l'agriculture et le développement.
}

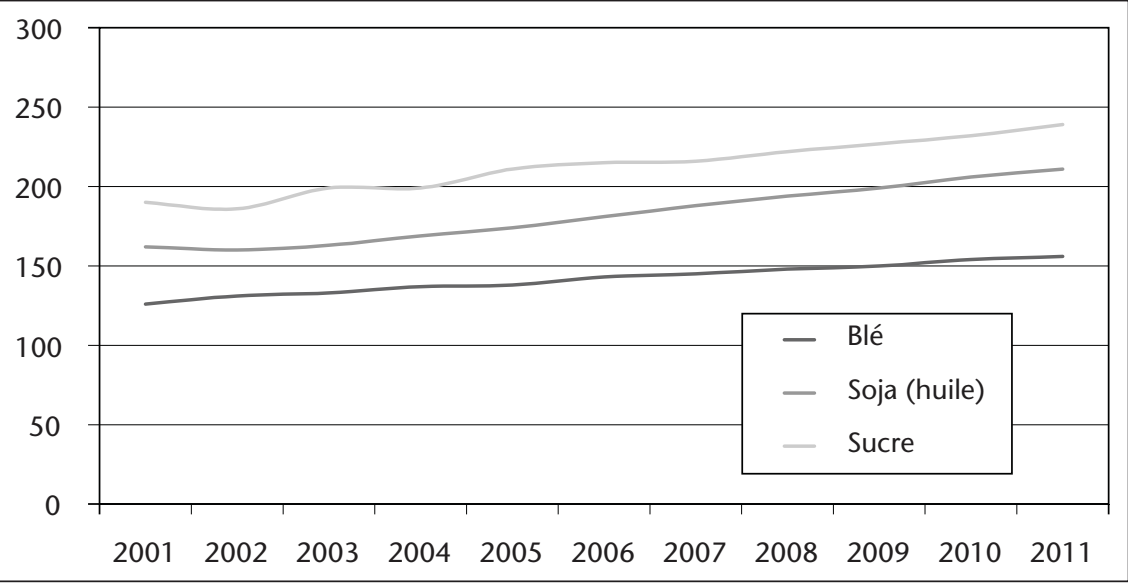

Figure 2. Prévision de prix dans FAPRI 2002 (US\$/bu). Source: FAPRI base line 2002. FAPRI (Food and Agricultural Policy Research Institute) est un consortium d'études économiques basé à lowa State University, à l'origine d'un modèle qui fait autorité (en dépit d'erreurs systématiques de prévision, cf. Baumel [4]).

peu de lumière sur cette question. Avant de le décrire et d'en donner les résultats, il faut cependant revenir sur les bases analytiques sur lesquelles il est fondé.

\section{Les explications alternatives}

Les modèles dont il vient d'être question sont basés sur la notion simple d'équilibre économique : il existe pour chaque produit une courbe d'offre et une courbe de demande. Si l'on est capable d'en donner une expression analytique, il doit être possible d'en chercher l'intersection, et de prévoir le prix qui va avec. Qu'il s'agisse, comme FAPRI, d'un modèle d'équilibre partiel (on considère que le niveau de prix obtenu pour le produit considéré ne modifie pas les revenus au point de modifier les paramètres des courbes $d^{\prime}$ offre et de demande), ou d'un modèle d'équilibre général (on tient compte de l'effet en retour des changements de prix sur l'ensemble des revenus et de tous les équilibres économiques du système considéré), la solution reflète toujours l'idée qu'il s'agit d'un équilibre stable qui, en général, correspond à l'égalité entre l'utilité marginale du consommateur et du coût marginal à long terme de production. Comme les coûts de production (eux-mêmes liés aux techniques) et les goûts des consommateurs varient assez lentement, ainsi font les prix, dont l'évolution dans le temps reste par conséquent relativement lente.

L'autre ligne d'explication est à rechercher dans le fonctionnement des marchés. Elle n'est pas nouvelle : on la retrouve chez Galiani [9] au $\mathrm{XVIII}{ }^{\mathrm{e}}$ siècle, qui prédisait que la libéralisation du commerce des grains allait entraîner la révolution, et, plus près de nous, chez les conseillers agricoles du Président Roosevelt qui furent les véritables théoriciens des politiques agricoles des années 40 à 90 [10]. Le cœur de leur analyse est que, en face d'une demande rigide, l'équilibre des marchés agricoles est essentiellement instable, comme celui d'une bille à la pointe d'un crayon : au lieu qu'un petit écart ramène le système à l'équilibre, il crée les conditions pour l'en éloigner encore plus ${ }^{5}$.

En l'espèce, la libéralisation conduite depuis les années 95, et accélérée depuis 2000, a provoqué une baisse de prix et une incertitude sur l'avenir qui a conduit les agriculteurs des pays développés à réduire un peu leur production. Venant après un délai de quelques années, cette baisse de production relativement faible, en face d'une demande fixe, provoque maintenant des hausses de prix très fortes.

La logique du modèle d'Ezekiel toutefois conduit à un résultat invraisemblable : plus on s'éloigne de l'équilibre, plus on est conduit à s'en éloigner encore plus! II faut donc qu'il

\footnotetext{
$\overline{5}$ Le modèle $d u$ « Cobweb » $d^{\prime}$ Ezekiel est fort simple, avec 3 équations pour trois inconnues : soient $q_{t}$ et $p_{t}$ les quantités et les prix au temps $t$ sur un marché défini par :

(1) $p_{t}=\alpha q_{t}+\beta$, (courbe de demande, $\alpha$ and $\beta$ en sont les paramètres, $\alpha$ est normalement négatif) ,

(2) $\hat{p}_{t}=a q+b$ (courbe d'offre, où $\hat{p}_{t}$ est le prix espéré pour l'année $t$, tandis que $a$ et $b$ sont des paramètres, avec a normalement positif). Alors, si en outre,

(3) $\hat{p}_{t}=p_{t-1}$.

le système "converge » vers un point d'équilibre stable si $\alpha / a$ est inférieur à 1 en valeur absolue, et « diverge » (les écarts à l'équilibre s'accroissent constamment au cours du temps) dans le cas contraire (on a mouvement périodique si par hasard $\alpha=-a$ ). Dans le cas des produits agricoles à demande rigide, $\alpha$ est grand en valeur absolue, tandis que l'offre est relativement "élastique », donc $a$ est petit. Les conditions sont donc réunies pour que le système diverge.
} 
existe un autre mécanisme capable de ramener le système en direction de l'équilibre quand on s'en éloigne trop: diverses hypothèses sont possibles en la matière, mais la plus simple et la plus naturelle est celle avancée par Wicksell en 1905 pour expliquer les cycles économiques: lorsqu'il existe une divergence trop évidente entre les coûts et les prix, les opérateurs deviennent conscients de ce que quelque chose d'anormal se produit, et, par des mécanismes plus ou moins complexes, cela ramène les prix vers les coûts de production [11]. Cette analyse, si elle est exacte, conduit à prédire que si les cours des produits agricoles augmentent aujourd'hui au-delà de toute raison, cette tendance va s'inverser au bout de quelque temps.

\section{Les preuves}

De fait, des modèles convenablement construits autour des idées précédentes, à la différence des modèles «standard » dont il a été question plus haut, permettent de reproduire le phénomène. $C^{\prime}$ est le cas du modèle $I^{3} \mathrm{du}$ CIRAD (Centre international de recherche agricole pour le développement) ${ }^{6}$. Celui-ci est construit comme un «modèle calculable d'équilibre général », à partir des données du consortium GTAP ${ }^{7}$ sur l'économie mondiale. Cependant, à la différence des modèles d'équilibre général, la production et la détermination des prix d'équilibre n'y sont pas simultanées. Les consommateurs sont approvisionnés par les récoltes de l'an dernier, tandis que les revenus sont distribués par les opérations de production de cette année, qui ne donneront de produit que I'an prochain. Les décisions de production sont donc prises sur la base de prix « anticipés » et non de prix d'équilibre, comme dans le Cobweb d'Ezekiel. En même temps, les producteurs savent que leurs anticipations ne se réaliseront pas forcément. Ceci les conduit à prendre des «primes de risque » et à calculer leurs coûts de production en conséquence. De même, les investissements en capital spécifique à chaque activité sont effectués sur la base des rentabilités constatées I'an dernier pour les différents types de capital, mais ici encore en tenant compte du risque associé à chaque décision $d^{\prime}$ investissement.

La figure 3 montre les résultats obtenus avec des données qui font partir la simulation de la situation qui existait dans le monde en 2001. Les trois courbes représentent le prix du blé en Union européenne dans trois scénarios ${ }^{8}$ : le premier (« base ») correspond à la poursuite de

\footnotetext{
${ }^{6}$ II est décrit en détail dans Boussard et al. [12] et Ayouz et al. [13]).

${ }^{7}$ Le consortium GTAP est une vaste entreprise de collecte de données et de construction de modèles sur l'économie mondiale. Cf Hertel et al. [14, 15].

${ }^{8}$ Ils sont décrits en détail dans Gérard et al. [16].
}

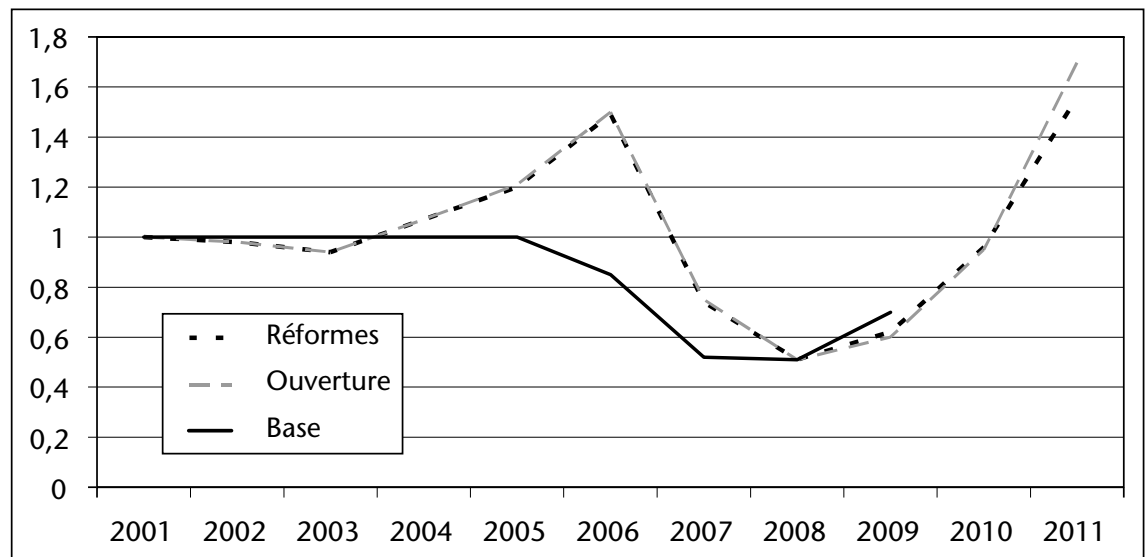

Figure 3. Indice du prix du blé dans trois scénarios (UE).

la PAC (politique agricole commune) de I'Union européenne dans le dispositif qui était en vigueur en 1999, avant la réforme de «l'agenda 2000 » de I'Union européenne. À cette époque, il y avait en particulier des « planchers " et des «maximums» de prix. Le second scénario (" réformes ») correspond à la situation crée par la réforme de la PAC en 2000 et 2003, et l'intensification du "découplage ». Le troisième enfin (« ouverture »), correspond, en plus des précédentes, à de nouvelles mesures de libéralisation douanières prises dans le cadre des accords envisagés à Doha, et à la suppression totale des subventions à l'exportation.

La figure 4 (extraite de Agreste, 2008) montre I'indice IPAP du prix du blé tendre de 2000 à 2008 : la parenté du début de la courbe « réformes » de la figure 3 avec la courbe des prix réels est frappante, même si le modèle ne décrit pas la petite hausse temporaire en 2003.

Il en est de même avec d'autres cultures dans d'autres parties du monde. Par exemple, le prix du sucre, avec ce modèle, tend à rester stable avec les politiques antérieures, il baisse avec la réforme de la $\mathrm{PAC}$ mais pour remonter prochai- nement, puis encore rechuter plus tard (figure 5)! Ces conclusions sont contraires à ce qu'attendaient beaucoup d'experts, mais conformes à la réalité jusqu'à maintenant (figure 6).

Ce modèle ne prend pas en considération les accidents climatiques, ni la croissance des agrocarburants, ni les comportements des "spéculateurs ». Pourtant, en se plaçant dans la situation de 2001, il projette dans le long terme une hausse des prix du même ordre de grandeur que celle que l'on observe actuellement. Cela donne confiance dans les résultats, et permet de penser que l'analyse sous-jacente - essentiellement, on l'a vu, le rôle des anticipations et du risque dans le comportement des agriculteurs - est correcte.

C'est pourquoi il semble possible de songer à s'en servir pour analyser l'impact des politiques agricoles mises en œuvre récemment ou envisagées.

Cela ne veut pas dire que l'accord est partout total - et ce fait même donne du crédit au modèle, qui n'a pas pu prendre en compte certains aspects des politiques agricoles survenus après son élaboration.

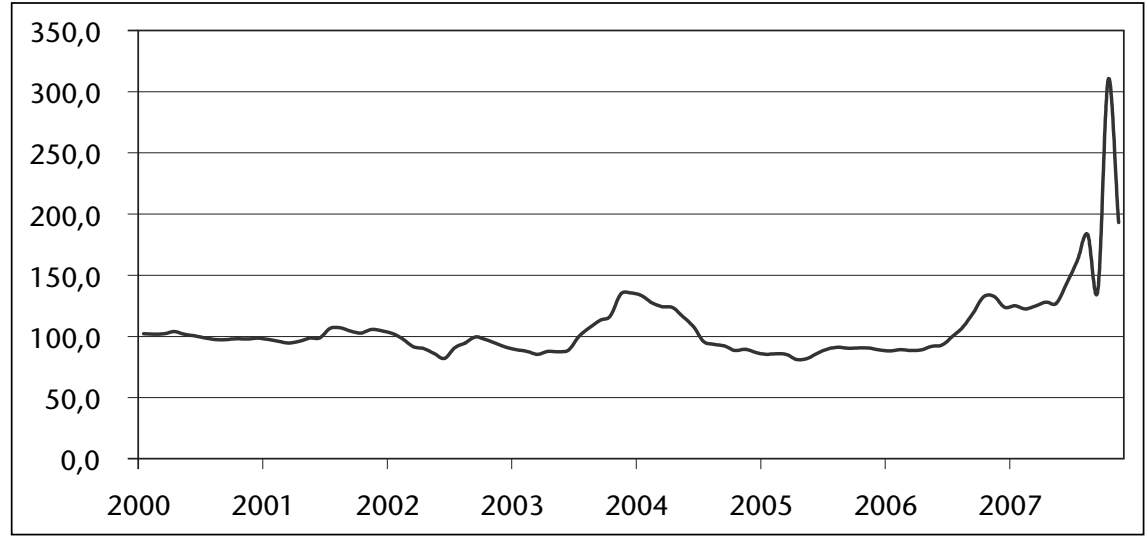

Figure 4. Indice du prix du blé tendre à la production en France (source: Agreste). 


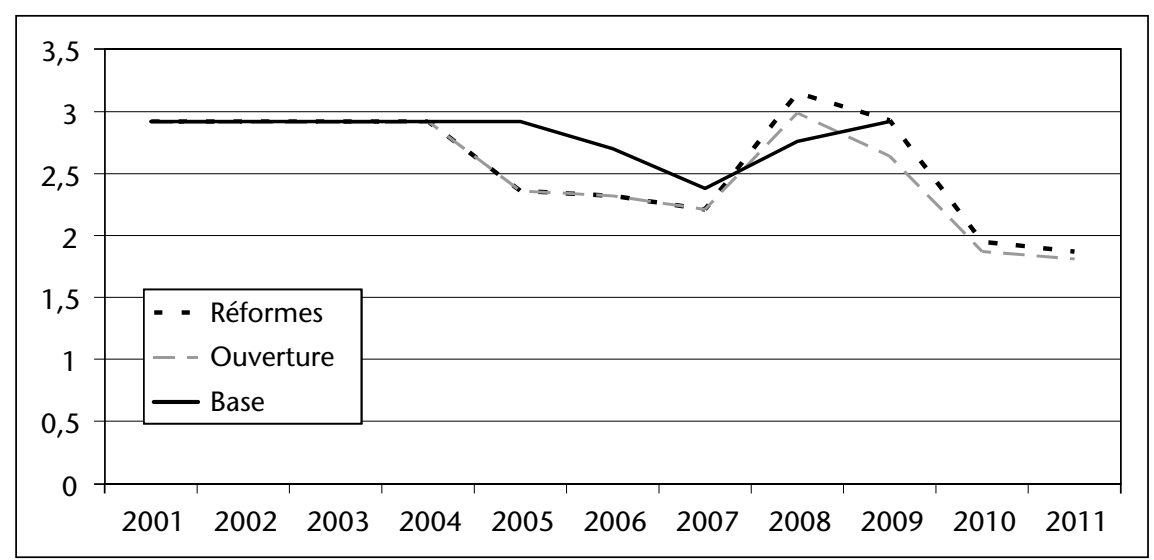

Figure 5. Indices du prix du sucre dans 3 scénarios (UE).

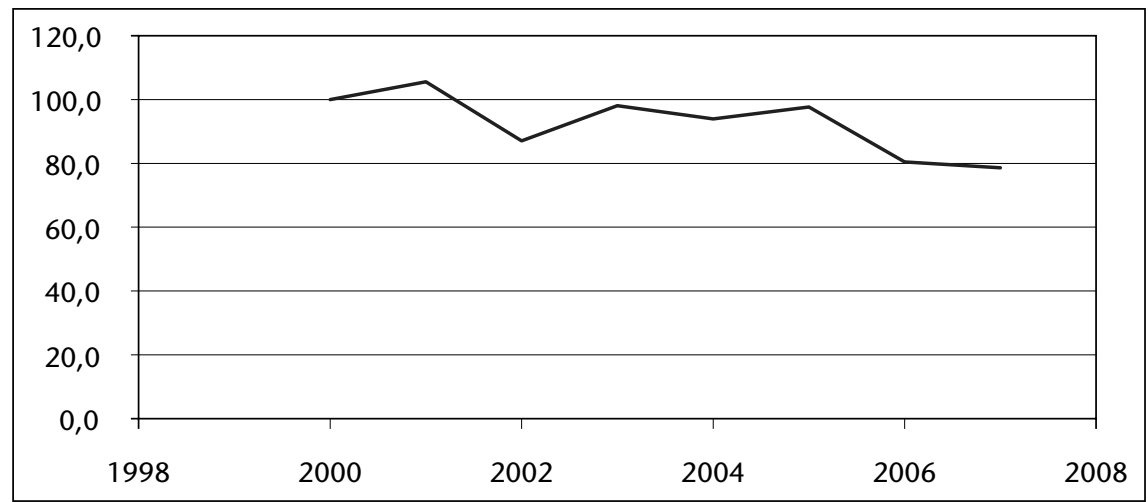

Figure 6. Indice du prix à la production des betteraves en France (source: Agreste).

Par exemple, la prévision est assez mauvaise pour les céréales secondaires aux USA, comme le montrent les figures 7 et 8 , qui donnent l'une les résultats du modèle pour les « autres céréales ", l'autre l'évolution réelle des prix du mais dans ce pays selon I'USDA (repris par la FAO). $\mathrm{Au}$ lieu $d^{\prime} u n e$ ascension progressive des prix jusqu'à un pic en 2006, la réalité des prix du maiis montre bien une ascension jusqu'en 2004, mais une dépression en 2005-2006, sui-

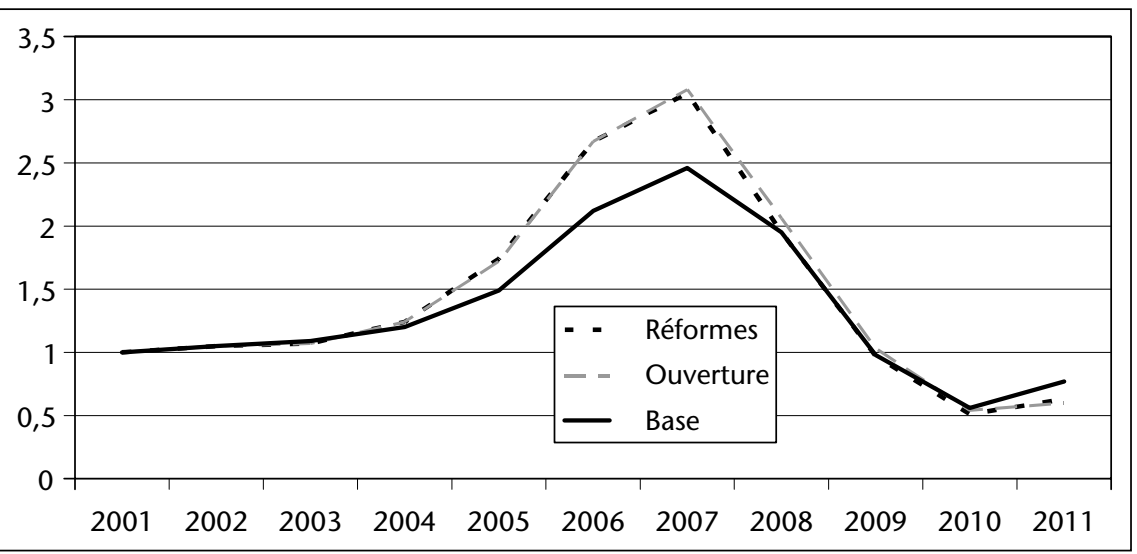

Figure 7. Indice de prix des autres céréales dans 3 scénarios (USA).
Globalement, cependant, et bien qu'il soit impossible de donner ici chacun des résultats un par un, l'accord entre le modèle ID3 et la réalité est presque surprenant, surtout si I'on prend en compte les nombreuses raisons pour lesquelles ce modèle aurait pu donner des résultats tout à fait différents.

\section{Quels effets pour combien de temps?}

\section{Les conséquences mondiales des politiques européennes}

Le modèle ID3 fourni des informations sur l'impact de chacune des mesures envisagées dans chacun des trois scénarios définis plus haut : poursuite de la PAC type 1999 (scénario « référence »), mise en œuvre de la PAC telle qu'elle a été définie en 2003 (scénario « réformes $")$ et intensification des réformes telles qu'elles sont envisagées dans le «bilan de santé » après 2008.

La conclusion majeure est qu'au lieu qu'il se produise en 2010, comme c'est le cas avec la situation "de base ", l'accélération des réformes de la PAC en 2003 a fait survenir le pic en 2006-2007 (situation « réforme »). C'est donc bien les réformes de la PAC en 2003 qui ont accéléré la hausse dans le reste du monde. Celle-ci, de toute façon, se serait produite vers 2010 si on était resté sur les scénarios issus de la réforme de la PAC en 1992 (et des réformes parallèles engagées dans les autres pays - en particulier les USA). A contrario, le scénario "ouverture», voisin de ce qui est envisagé pour la prochaine réforme de la PAC, ne produit presqu'aucun effet.....

Le changement principal introduit par la réforme de la PAC dans ces scénarios est le fait qu'alors que les agriculteurs peuvent baser leurs décisions de production sur un prix qu'ils sont à peu près sûrs de recevoir, la réforme les met dans une situation plus incertaine, ce qui les conduit à réduire les volumes offerts : c'est pourquoi la production baisse et les prix augmentent ${ }^{9}$.

Ce n'est donc pas les événements météorologiques qui ont provoqué cette crise, mais la réaction logique des producteurs aux messages qui leur ont été transmis par le marché. On voit en même temps que les politiques de libéralisation engagées depuis une vingtaine d'années ont joué avec le feu, en induisant des comportements excessifs, comme ceux que les politi-

\footnotetext{
${ }^{9}$ En fait, sensiblement plus qu'en réalité. Cela est compensé par une réactivité de la demande au prix sans doute plus forte qu'en réalité, qui atténue l'effet amplificateur de la rigidité de la demande sur les variations de prix entraînées par un choc sur l'offre.
} 


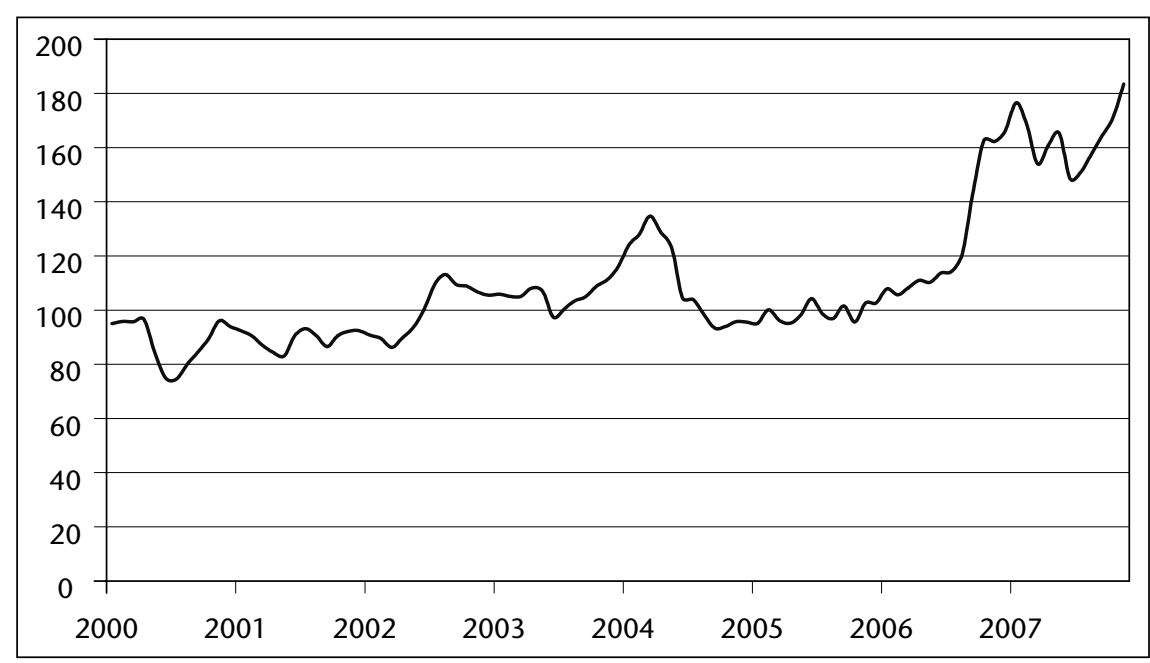

Figure 8. Prix du maïs aux Etats-Unis, $\$ /$ bush (source : USDA).

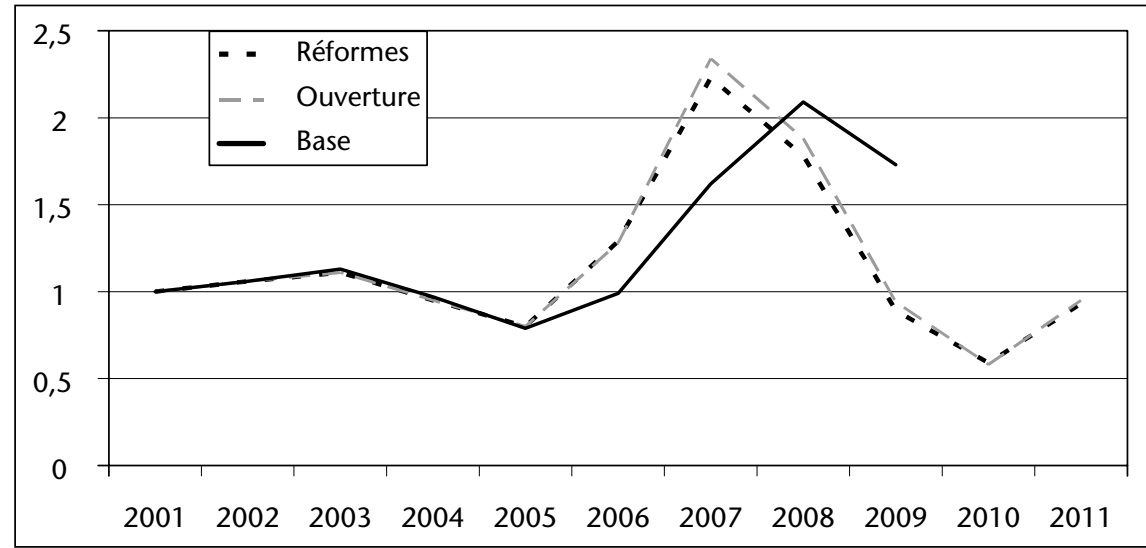

Figure 9. Indice du prix du lait dans 3 scénarios (Chine).

ques de contrôle édifiées depuis les années 30 avaient justement cherché à corriger. On voit aussi que les politiques énergétiques - en réalité, un retour en arrière vers des politiques " industrielles » comme il y en avait dans les années 60, avec mise en avant de l'argument d'indépendance économique, mais visiblement mal coordonnées avec les politiques agricoles - ont pu jouer le rôle d'adjuvant dans la genèse de la crise, sans cependant en être l'origine première.

On voit de même que les politiques récentes ont pu accélérer les événements sans cependant en changer la physionomie. Cela est évident quand on regarde les figures 3 et 5 , qui montrent que les réformes ont provoqué vers 2007 une élévation de prix qui se serait de toute façon manifestée vers 2010. En réalité, en 1999, la PAC conservait encore de nombreux mécanismes de type « filets de sécurité » qui en faisaient un « stockeur en dernier ressort » pour beaucoup de produits agricoles. De ce point de vue, elle avait remplacé les USA qui avaient joué ce rôle dans les années $70^{10}$ Ces mécanismes étaient coûteux pour le pays qui jouaient ce rôle, mais, en stabilisant les prix, ils étaient très bénéfiques pour le reste du monde. C'est pourquoi du reste l'Union européenne s'était trouvée plus ou moins contrainte de prendre le relai des USA lorsque ceux-ci ont refusé de jouer ce jeu. Mais ce n'était pas de tout cœur, et les réformes successives, depuis 1992, ont pour l'essentiel consisté à se décharger progressivement de ce fardeau. Même si, en 1999, une partie en avait déjà été évacuée, il en restait encore assez pour retarder la crise au moins jusqu'en 2010. La réforme de 2003 a supprimé les derniers vestiges des politiques de régulation antérieures. Elle a provoqué la crise beaucoup plus tôt. II semble que l'on soit arrivé maintenant à un point où une libéralisation encore plus forte ne sera plus susceptible de produire aucun effet, du moins sur les prix et les

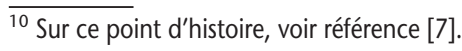

productions: c'est le sens de la similitude des courbes relatives aux scénarios « réformes » et " ouverture» :

Enfin, il ne faut pas sous-estimer l'impact des politiques sur le reste du monde. Par exemple, la figure 9 représente l'impact des trois scénarios sur le prix du lait en Chine. La politique européenne a légèrement aggravé la situation de la Chine en matière de produits laitiers : en fait, elle a avancé un pic de prix qui se serait de toute façon produit un peu plus tard. On voit bien aussi que la hausse du prix du lait en Chine (et sans doute aussi ailleurs) ne sera que temporaire $^{11}$.

\section{Cela va-t-il durer?}

II serait fastidieux de poursuivre l'énumération de ces graphiques qui se ressemblent. II faut maintenant tirer les leçons de l'expérience réalisée avec ce modèle.

La hausse va-t-elle durer? Sur la base de ce modèle, la réponse est non: les prix vont retomber au bout de quelques années, du fait de la croissance trop importante de l'offre suite à la flambée des prix. Ils devraient retomber plus bas qu'avant, entraînant sans nul doute la faillite d'un grand nombre d'agriculteurs, préparant ainsi une hausse ultérieure encore plus forte ${ }^{12}$.

En réalité, ce modèle, si fascinant soit-il, ne fait que reproduire les résultats qualitatifs obtenus par Boussard [10] : du fait de l'instabilité de son point d'équilibre, un marché de matières premières à demande rigide est nécessairement chaotique (au sens mathématique du terme), et doit donc fluctuer de manière considérable, de manière imprévisible à long terme (même si des prévisions à court terme sont possibles, comme pour le temps qu'il fait). II reste à savoir au bénéfice de qui.

\section{Gagnants et perdants}

Le modèle effectuant la distinction entre «ménages riches » et «ménages pauvres», il

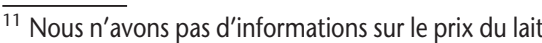
en Chine pour vérifier la conformité de cette projection avec la situation réelle. On trouve cependant sur Internet des informations selon lesquelles les prix du lait, après avoir fortement monté en 2006, seraient restés sensiblement constants ou en légère décroissance en 2007.

${ }^{12}$ On pourrait penser que cette baisse prévisible sera moins grande qu'indiquée ici, à cause des biocarburants, qui ne sont pas inclus dans ce modèle. Si cela se confirmait, il faudrait par la suite envisager une nouvelle hausse encore plus grande. D'une façon générale, on ne voit pas que les biocarburants puissent avoir un rôle stabilisateur, bien au contraire, s'ils sont soumis à la loi de l'offre et de la demande.
} 


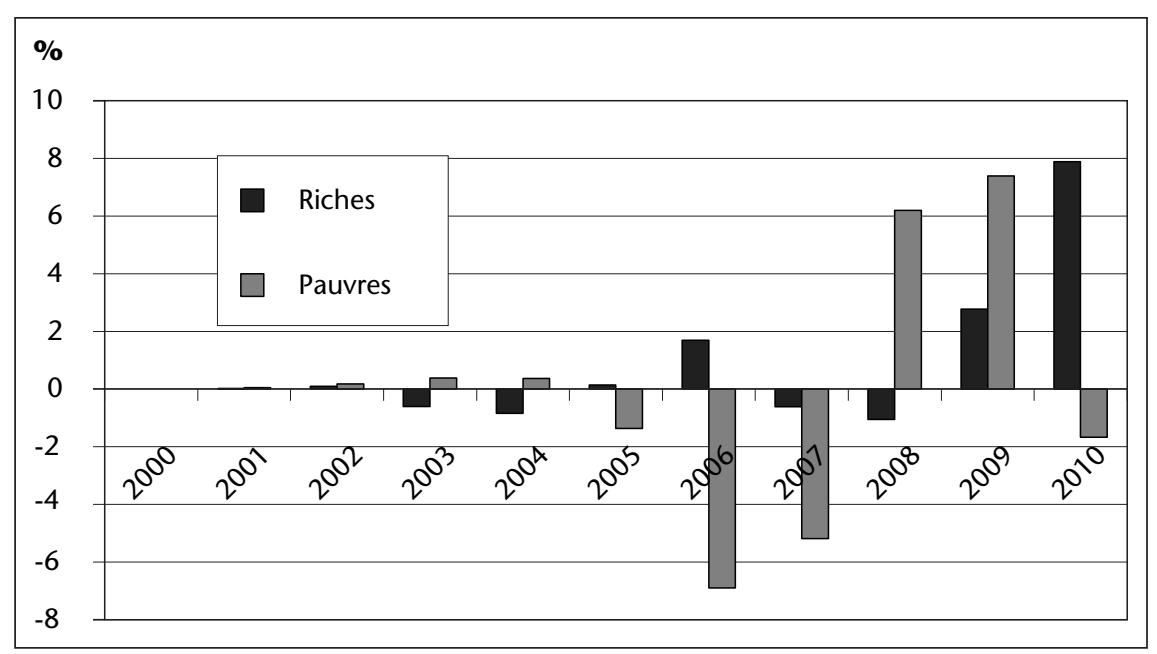

Figure 10. Accroissement d'utilité des ménages entre les deux scénarios «base " et " ouverture " dans les PMA (\% base).

était tentant d'en examiner les résultats sous l'angle des «niveaux d'utilité » correspondants.

II ne faut pas se faire d'illusions sur la précision de ces résultats: en l'absence de distinction entre « riches » et « pauvres » dans les données de la base GTAP utilisées, il a fallu des hypothèses héroïques pour obtenir des résultats en la matière. Elles sont décrites chez Boussard et al. [12]. En fait, les revenus des riches dépendent pour l'essentiel (mais non exclusivement) des revenus du capital, tandis que ceux des pauvres dépendent du travail. Par ailleurs, les « niveaux d'utilité » indiqués dépendent aussi de l'hypothèse selon laquelle la demande des ménages serait déterminée par une fonction de type " Linear expenditure système » ce qui est fort discutable. Enfin, on a négligé les évolutions démographiques, comme les changements liés au progrès technique, ce qui est aussi discutable $^{13}$.

La figure 10 montre cependant, en dépit de ces réserves, que les bénéfices à attendre de ces réformes sont au moins inégaux. Ce qu'il y a de plus frappant, c'est que, au jeu de la libéralisation, tantôt les uns tantôt les autres perdent ou gagnent sans qu'il soit possible de discerner une règle générale.

Cela s'explique par l'ambigüité des effets des hausses ou des baisses de prix alimentaires: comme il y a beaucoup de pauvres à être agriculteurs, en principe, les hausses de prix les avantagent. Mais ils sont aussi consommateurs, et, de ce point de vue, les hausses de prix leur sont pénibles. Les riches, en revanche, sont peu

\footnotetext{
${ }^{13}$ On peut évidemment supposer que ces deux facteurs jouent en sens inverse et au même rythme, s'annulant ipso facto. Tout de même, il s'agit ici encore d'une hypothèse héroïque.
}

sensibles aux hausses de prix, dans la mesure où leurs consommations alimentaires pèsent moins dans leurs budgets. D'un autre côté, percevant moins de revenus d'origine agricole, ils n'ont que peu d'accroissement de revenus à attendre d'une hausse des prix.....

Quoi que ce ne soit pas montré par le modèle, qui n'intègre pas les mécanismes de développement de l'innovation ni les modifications de nécessaires pour le mettre en évidence, il est cependant permis de penser que, en réalité, tout le monde perd à ce jeu de yoyo des prix agricoles. Cela tient à deux choses. D'une part, les hausses de prix, même temporaires, sont extrêmement coûteuses en perte d'utilité pour les ménages pauvres non-agriculteurs ${ }^{14}$. D'autre part, l'incertitude sur les prix freine le progrès technique dans des proportions qui pour n'être que difficilement chiffrables, sont probablement considérables. À long terme, par conséquent, c'est véritablement l'ensemble de I'humanité qui pâtit de ces dysfonctionnements des marchés.

\section{Conclusion}

Les conclusions précédentes reposent évidemment sur la validité du modèle ID ${ }^{3}$, par opposition à celles que l'on aurait tiré du « modèle tatés ces derniers mois relèvent du simple hasard climatique, ou de décisions malheureuses mais ponctuelles, comme celles qui ont trait aux biocarburants (étant entendu que la crois-

\footnotetext{
${ }^{14}$ Malthus n'affirmait-il pas que l'équilibre entre I'offre et la demande alimentaire se faisait grâce à la mortalité des pauvres ? Ne serait-ce pas encore le cas aujourd'hui?
} régimes démographiques qui auraient été standard ", selon lequel les phénomènes cons- sance des pays émergents devrait avoir été prévue - en tout cas, envisagée - par les modèles d'équilibre général).

Bien entendu, $I D^{3}$ n'est pas un modèle de prévision. En particulier, les pics peuvent être décalés dans le temps, et se révéler plus ou moins marqués que ce qui est envisagé ici. $D^{\prime}$ une façon générale, il est toujours difficile d'évaluer le pouvoir prédictif d'un modèle à plusieurs dimensions: que veut dire la bonne adéquation des prévisions pour le sucre s'il existe un énorme écart pour le blé ? Cette question de la validation est récurrente dans toutes les activités de modélisation économique lorsque le modèle vise à reproduire le comportement d'un grand de variables simultanément.

II est sûr aussi que ce modèle souffre d'une réactivité un peu forte de la production au prix, entraînant des baisses de production plus importantes que la réalité, partiellement compensées par une élasticité de la demande sans doute plus forte que ce qui existe réellement. Mais presque tous les modèles de ce type souffrent de ce genre de défauts.

II se trouve cependant qu'il a très bien fonctionné (dans l'incrédulité générale) fin 2005 pour prédire la crise actuelle, ce qui lui donne une certaine crédibilité. Même s'il ne faut donc pas prendre ses prévisions au pied de la lettre, on peut lui attribuer une certaine valeur qualitative : certainement, les très fortes hausses de prix que nous observons actuellement ne sont pas le fait du hasard, elles sont la conséquence de la dérégulation des marchés agricoles depuis les années 1990, et elles ont été accélérées par les réformes de la PAC ces dernières années.

Ainsi, et contrairement aux hypothèses « standard » en la matière, la dérégulation des marchés agricoles ne met pas à l'abri de crises, et ne réduit pas forcément l'instabilité des prix. Or cette instabilité est susceptible de contrecarrer les bénéfices à attendre d'un meilleur jeu des avantages comparatifs suite à la libéralisation des échanges. Il est sans doute important d'en tenir compte dans les réformes de politiques agricoles.

\section{RÉFÉRENCES}

1. AGRESTE-CONIONCTURE. Editorial. Panorama, Juillet 2007.

2. OCDE-FAO. La croissance de la demande de biocarburants alimente la hausse des prix agricoles. Rapport conjoint, Paris-Rome, 4 juillet 2007.

3. VON BRAUN I. The world food situation : new driving forces and required actions. Washington : IFPRI, 2007. 
4. BAUMEL CP. How U.S. Grain Export Projections from Large Scale Agricultural Sector Models Compare with Reality. Prepared for the Institute for Agriculture and Trade Policy, Minneapolis, MN May 292001.

5. PREBISCH R. Toward a New trade Policy for Development. New York : United Nation, 1964.

6. SINGER $\mathrm{H}$. The distribution of gains between investing and borrowing countries. Am Econom Rev $1950: 40$.

7. BERTRAND JP, DELORME H. Pratique de la régulation des marchés agricoles internationaux: le cas du blé et du soja. In : Boussard et Delorme, 2007.
8. GERONIMI V. Les cours internationaux des produits agricoles : tendances et cycles. In : Boussard et Delorme, 2007.

9. GALIANI F. Dialogue sur le commerce des bleds. Paris : 1770 ; Réédition, Fayard, 1984.

10. EZEKIEL M. The Cobweb Theorem. Quaterl I Econom $1938 ; 53$ : 225-80.

11. BOUSSARD JM. When risk generates chaos. J Econo Behav Organ 1996 ; 29(96/05) : 433 46.

12. BOUSSARD JM, GÉRARD F, PIKETTY MG. Libéraliser l'agriculture? Théorie, modèles et réalité. Paris : Editions Quae, 2005.
13. AYOUZ M, BOUSSARD JM, GÉRARD F, PIKETTY MG, VOITURIEZ T. Endogenous risk and long run effects of liberalization in a global analysis framework. Econom Model 2006; 23(3).

14. HERTEL T, ET AL. Global trade analysis. Cambridge : Cambridge University press, 1997.

15. HERTEL T, KEENEY R, VALENZUELA E. Global analysis of agricultural trade liberalisation :Assessing model validity. Paper presented at the AAAE meeting, Denver (Colorado), June 1-4. 2004.

16. GERARD F, PIKETTY MG, BOUSSARD JM. Analyse de l'impact de scénarios de libéralisation des échanges agricoles internationaux à partir $d u$ modèle $I D^{3}$. Paris: Rapport au Ministère de I'Agriculture, 2008. 